Title

From milliseconds to lifetimes: Tracking the dynamic behavior of transcription factors in gene networks

Ying $\mathrm{Li}^{1}$, Kranthi Varala ${ }^{1}$ and Gloria Coruzzi ${ }^{1}$

${ }^{1}$ Center for Genomics and Systems Biology, Department of Biology, New York University, New York, NY 10003, USA.

Corresponding Author: Dr. Gloria M. Coruzzi, Center for Genomics and Systems Biology, Department of Biology, New York University, New York, NY 10003, USA. (212) 998-3963. gloria.coruzzi@nyu.edu

\author{
Keywords \\ Gene regulatory networks; transcription dynamics; transcription factors
}

\begin{abstract}
Modeling dynamic gene regulatory networks (GRNs) is a new frontier in systems biology. It has special implications for plants, whose survival requires rapid deployment of GRNs in response to environmental changes. However, capturing and dissecting transient interactions of transcription factors (TFs) and their targets in GRNs remains a considerable experimental challenge. Here, we review recent progress on understanding GRNs as a function of time, and discuss the relevance of these findings in plants to studies in other eukaryotes. We cover progress in profiling and modeling time-course transcriptome changes across plant species, and the insights they have provided into the regulatory mechanisms underlying these temporal transcriptional responses, with a focus on the dynamic behavior of TFs. Lastly, we review state-of-the-art techniques to monitor single-molecule dynamics of TFs in vivo. Together, these advances have helped develop new models for dynamic transcriptional control with relevance across eukaryotes.
\end{abstract}

\title{
Transcription in real time
}

Modeling the dynamics of gene regulatory networks (GRNs) (Box 1; Fig. 1) requires the ability to experimentally capture interactions between transcription factors (TFs) and their genome-wide targets as a function of time. At present, this is an experimentally formidable but illuminating task. Although the timely transcriptional regulation of gene networks is of critical importance to the survival of all living organisms, it is especially so for plants that must coordinate a rapid transcriptional response to environmental and developmental cues, making them an ideal model for studying dynamic GRNs.

In recent years, there have been significant technological advances that have enabled large-scale investigations into gene regulatory network responses with increasing temporal and spatial resolution. Decreasing sequencing costs have led to widespread profiling by RNA-Seq and ChIPSeq data in real time. This has enabled studies of TF-target binding assayed in response to environmental stimuli and during development. Here, we first review recent progress in profiling and modeling genome-wide expression changes across various plant species, with time-scales ranging from minutes to days (Fig. 2A). We next discuss new insights into the regulatory mechanisms underlying these transcriptional responses with a focus on the dynamic behavior of TFs. More broadly, we highlight how these findings in plants relate to recent studies in yeast and animals. Finally, we review the latest state-of-the-art approaches to monitor single-molecule 51 dynamics of TFs in vivo through live-cell imaging (Fig. 2 B\&C) [1,2]. The net result of these 
advances in real-time expression profiling, network discovery, and single molecule dynamics is the development of new models for transcriptional control, such as the hit-and-run [3] and molecular clutch models [4], with relevance across eukaryotes.

\section{Dynamic GRNs}

Increasing access and decreasing costs of next-generation sequencing technology in the past decade have led to a dramatic rise in time-series assays of genome-wide transcriptional responses. These studies, conducted over minutes, hours, or days (Fig. 2), have identified distinct temporal gene expression patterns for different genes in a gene regulatory network, including stably and transiently up- or down-regulated gene expression patterns (Fig. 2A). In plants, it has been shown that the response to abiotic or biotic stimuli occurs from minutes to hours, enabling survival in a rapidly changing environment. These temporal responses of dynamic gene networks (where the "nodes" (genes) shows changing expression over time) [5-7], are largely triggered by key TFs.

One example of this is nutrient sensing, where the TF SPL9 drives gene network responses as rapidly as 3 minutes after root exposure to nitrate [5,8]. This nitrate-induced gene network responses include transient induction, consistent induction and transient/stable repression of different gene clusters [5]. In the case of light exposure, the master TF PIFs are critical for achieving a transcriptomic response within one hour of the stimulus [9]. In contrast to these rapid responses to abiotic signals measured in $\leq$ one hour, the response to biotic signals such as pathogen attacks are usually measured over the course of multiple hours [10]. This is likely due to the nature of pathogen infection - that it is difficult to measure response in minutes unless single cells or protoplasts are challenged with pathogen. In one study [10], the transcriptomic response to a biotic stress is mostly through transient gene regulation, where gene expression increases in the first 12-18 hours and goes down to the normal level by 24 hours [10]. This transient transcriptional response observed across hours may suggest a control by multiple feed-back loops to restrain the scale and scope of the defense response. Finally, stable or transient up- or downregulated genes are also observed over developmental time, usually measured in days (Fig. 2A), in a range of processes such as flowering [11], endosperm development [12,13], fruit ripening [14], and seed development [15]. These temporal expression patterns in development can be driven by $\mathrm{TF}$ regulation [12] or epigenetic regulation such as imprinting [13], or both. Importantly, plant development is heavily influenced by environmental factors, such as light [16], nutrient [17,18], and gravity [19], indicating that even when a GRN is carrying out a long-term developmental program, it must also respond dynamically on shorter time-scales.

Although plants are particularly susceptible to changing environmental conditions, the temporal behavior of GRNs in response to developmental cues and environmental changes seems to be shared across kingdoms, and likely reflects a basic nature of gene networks. For example, transiently and stably regulated gene expression patterns were also reported in mammalian cells in response to pathogen challenge [20] and in yeast in response to salt stress [21]. Although many studies report these temporal gene expression patterns driven by developmental or environmental cues, less is known about the underlying regulatory mechanisms, especially in plants. It is clear, however, that transcriptional control is extensively mediated by TFs [5,12]. Thus, examining the temporal behavior of TFs genome-wide is often the starting point to further explorations of dynamic GRNs.

\section{Global dynamic patterns of TF binding}

Transcriptional responses are governed by TFs that bind the specific cis-regulatory sequences in the promoter of target genes. As such, identifying when and where a TF binds to target sites in the 
genome can provide insights into the architecture of the transcriptional response. A study of mesoderm development in Drosophila [22] evaluated the deterministic power of temporal interactions of TFs and their target genes on the regulation of a gene network. After monitoring the global DNA binding pattern of five TFs over multiple stages during mesoderm development, the resulting TF-target binding data was used to train a machine-learning algorithm to predict the spatio-temporal expression pattern driven by the TF-bound promoters. Surprisingly, with only the stage-specific TF-binding data as inputs, the model can successfully predict the spatio-temporal gene expression at an accuracy of $>70 \%$. This mesoderm development study in Drosophila thus suggests that the time-specific TF interactions with their targets are a major deterministic factor of temporal gene expression patterns.

A recent study of EIN3, the master TF regulator of the ethylene response in plants, revealed important features of TF regulation of GRNs as a function of time [23]. EIN3 binds to its genomic targets in response to ethylene treatment. However, EIN3 binding does not necessarily lead to a change in target gene expression, as only $30 \%$ of the EIN3-bound genes showed a change in expression level during the ethylene treatment [23]. For many EIN3 bound- and ethylene-responsive genes, the binding by EIN3 peaked at four hours after the ethylene treatment, and the binding measurably weakened after 12 and $24 \mathrm{hrs}$ [23]. Interestingly, the expression level of these EIN3 target genes forms four distinct waves following ethylene treatment [23]: One wave shows an immediate increase within as soon as $15 \mathrm{~min}$ after ethylene treatment. This wave probably represents the early responders. The other three waves of EIN3 target genes show later responses $(>1 \mathrm{hr})$ with differing slopes of ascent. Remarkably, all the EIN3 regulated target genes show a steady increase of expression level during the full course of the $24 \mathrm{hr}$ treatment, even after EIN3 binding weakens after 4hrs [23]. Therefore, EIN3 may start the transcriptional activation of the target genes, and leave after a few hours, while the transcription is likely maintained by other factors. The observation that similar binding patterns lead to distinct transcriptional outputs (expression change vs. no change, and different patterns of expression changes) may reflect additional impacts of TF partners, chromatin structure, and post-transcriptional regulation that modify the output of TF binding.

Although the above studies and other ChIP-based whole-genome profiling studies are a rich source of information about the modes of regulation of specific TFs [23-26], they are difficult to extend beyond one or a few TFs in a single experiment. Recently, an alternative approach, DNase I sequencing, was used in Arabidopsis to identify the genomic TF-binding footprints of multiple TFs in response to light [27]. By sequencing DNase I fragments at great depth, this approach identified DNase I hypersensitive sites (DHSs), and the TF footprints within these sites, to delineate TF occupancy. The photodynamic DHSs were shown to be clustered into five temporal patterns reflecting distinct dynamic interactions of TFs with their targets: stably down, early transient up, late transient up, early stably up, and late stably up. A TF network was constructed based on DNAse I footprints, and dynamic rewiring of this network was observed upon the exposure to light. Importantly, this TF network in Arabidopsis shares similar basic motifs (see Box 1) with those identified in human or C. elegans networks [27].

Taken together, these studies share a common observation that the binding of TF to targets changes overtime and in response to stimuli $[23,27]$. How is this regulated? One possible mechanism to modulate the timing of TF-target interactions is by regulating the nuclear localization of TFs. This was recently described for NLP7, a master TF that mediates nitrogen responses in plants. NLP7 stays in the nucleus when nitrate is present, otherwise it is exported out of nucleus [28]. This nuclear localization control allows NLP7 to regulate global gene expression in response to nitrate, within minutes of a supply of nitrate [28]. 
1

What is the biological relevance of these dynamic behaviors of TFs, e.g., their binding and dissociation from their targets? A recent study in Arabidopsis focused on bZIP1 [3], a ubiquitously expressed TF associated with nutrient signaling across eukaryotes [29-31], suggests that transient bZIP1 binding specifically mediates rapid responses to a nutrient stimulus in plants [3]. This study identified transient targets bound by bZIP1 within minutes after bZIP1 enters the nucleus, where the transcription of the target gene initiated by the TF "hit" can still be observed when the TF is no longer bound (e.g. at $5 \mathrm{hr}$ ). This finding for bZIP1 is similar to the study of the EIN3 TF, whose binding to gene targets peak at $4 \mathrm{hrs}$ and is weakened by $24 \mathrm{hrs}$, while the ethylene induced gene expression response keeps increasing between 4 and $24 \mathrm{hrs}$ [23]. This transient TF binding of bZIP1 named "hit-and-run", specifically activates the transcription of key genes involved in rapid nitrogen signaling and nitrate uptake in roots [3,5], thus offering a functional explanation of the transient binding behavior of a TF (Box 2). This "hit-and-run" mechanism observed at the genome-wide level for bZIP1, was also previously implicated for individual genes in mammalian cells [32-34]. Based on the genome-wide data for bZIP1, this mechanism likely enables a small number of TFs to act as "TF catalysts" to activate a large gene network for an acute response to a signal.

The continued transcription of the transient targets of bZIP1 even after bZIP1 disassociates from the promoters is possibly maintained by the recruitment of other regulatory factors $[3,34]$. Supporting this hypothesis, the promoters of transient targets of bZIP1 are enriched with cis binding motifs for other TF families such as MYBs [3]. Similarly in humans, it was suggested that TFs are likely to be frequently recruited as co-factors by other TFs based on a compilation of ENCODE [35] ChIP-Seq data for 96 TFs from 5 human cell lines [36]. It might be important for genes involved in response to stimulus to be co-regulated by multiple TFs, so that the gene regulatory network can integrate signals from multiple channels. Indeed, a comprehensive analysis of global binding profiles of 27 plant TFs found that target genes that are co-regulated by multiple TFs are significantly enriched for genes responsible for stimulus response and signaling [37]. Studies on the light signaling hub - the PIF family, revealed a complicated network where the four PIFs direct differential light signaling to the down-stream transcriptional network, each PIF has different impact on the transcriptional output of shared targets $[38,39]$. Combinatorial TF effect may explain why the same TF binding pattern could lead to different transcriptional outputs, as reported for Arabidopsis TF EIN3 [23]. In this case, the different EIN3 targets might be coregulated by different TF partners, which lead to difference in temporal gene expression pattern.

Indeed, these recent findings on dynamic TF binding in plants $[3,23,27]$ have parallels in yeast and animals [20,21]. Overall, different TFs can display different dynamic behaviors, and a specific TF can have distinct dynamic binding patterns at different targets, which partially accounts for the temporal expression pattern of the targets. For example, salt response in yeast was used as a model to study dynamic TF binding in regulating complex biological processes [21]. This yeast study revealed distinct classes of temporal TF binding for a couple of TFs, including constant binding, rapid induction, delayed induction, transient increase or decrease, or a gradual increase in binding. These temporal TF-binding patterns are similar to the ones revealed by DNase I sequencing in Arabidopsis described above [27]. Meanwhile, gene expression during the yeast salt response shows three major patterns: constitutively expressed, transiently induced, and transiently repressed. This clustering of dynamic gene expression patterns in yeast [21] is comparable to those observed in plants [5,14]. An integrated study of the dynamic binding and expression data from yeast [21] suggests that a specific type of dynamic TF binding pattern can lead to different temporal patterns of gene expression. This is in agreement with the plant TF EIN3 [23] and suggests that other factors play roles in determining gene expression patterns, e.g., TF partners or chromatin modification. Nonetheless, some specific associations of a TF binding pattern and an expression pattern can be observed. For example, the rapid binding of the TF Yap4 
correlates with a transient increase in gene expression. The temporal binding patterns of multiple TFs assayed in this study [21] allowed the construction of a model of a dynamic transcription network in yeast.

Finally, a high-throughput temporal TF binding dataset from mammalian cells further revealed principles of dynamic gene regulation [20]. This study measured the dynamic DNA binding of 25 TFs at four time-points following pathogen stimulus of primary innate immune dendritic cells. As was observed in yeast and plants, the TFs ranged from static binders to dynamic binders. An interesting observation from this simultaneous multi-TF study is that many of the defense-related TF binding events are observed before pathogen stimulation, predominantly on immediate early responsive genes. The TF binding prior to the abiotic stimulus was also observed in the salt stress study in yeast [21], where constitutive TF binding possibly primes target genes for immediate reaction. Gene targets in the inflammatory response pathway were also found to be more likely bound by multiple TFs. This finding shares similarities with the Arabidopsis bZIP1 study in that the targets co-regulated by bZIP1 and other TF partners are specifically relevant to nutrient responses in root cells [3]. The poised TF binding [20] and the regulation by multiple TFs [3,21] suggest that GRNs are primed to respond to an external stimulus and accept input from multiple TF sources.

Overall, many parallels can be drawn between these gene network studies in plants and animals, which collectively provide a dynamic view of GRNs. By contrast to a static image, the GRNs changes over time, likely driven by the time-specific interactions between TFs and targets. Transcriptomic studies in both plants and animals have shown various temporal gene expression patterns for different genes in the network, with some genes transiently regulated and others consistently regulated. Dynamic TF binding to the target promoters were also reported across systems, for example, one TF can have stable or transient binding on a gene-by-gene basis [3,21]. The projections from the temporal TF binding to the temporal gene expression pattern in a GRN are rather complicated and possibly involve multiple layers of regulation. However, studies from plants and animals reach some common conclusions on this matter: the same temporal TF binding pattern can lead to different gene expression outputs for different target genes, suggesting other factors such as chromatin modification or TF-cofactors also play a role [3,20-22,37]; nonetheless, in some cases temporal TF binding patterns can be predictive of gene regulation patterns $[21,22]$. Finally, networks in plants and animals contain shared basic motifs [27]. These studies reveal conservation in the dynamic behavior of gene regulatory network across eukaryotic systems. Interestingly, the animal and yeast studies are typically performed in cell-lines or single-cell setups, raising the question whether the observed GRN behavior will be the same in a multicellular organism. Therefore, the plant studies performed in whole organism help validate the in vivo relevance of these general findings in the context of a multiple cellular organism.

\section{Dynamic behavior of individual TFs in the nucleus}

The studies covered in this review so far typically measure TF binding using biochemical methods such as ChIP or DNase I, followed by next-generation sequencing. The binding kinetics is therefore determined for a population of TF molecules, rather than for individual TF molecules. For example, if the TF binding is detected by ChIP at 5 and $30 \mathrm{~min}$, does that mean the same TF molecule stays on the promoter that whole time, or does it dissociate and another molecule binds again? It could also reflect that the target is bound at 5 minutes in one cell and in another cell the target is bound at 30 minutes. Some recent studies are moving beyond this limitation and starting to shed light on these questions. 
In a yeast study, competition ChIP-chip was used to detect the binding kinetics of Rap1 [4]. The unique feature of this ChIP experiment was that Rap1 was labeled using two different tags, which enables the measurement of turnover rate of Rap1 at a promoter, i.e. how long it takes for Rap1 with tag A to be replaced by Rap1 with tag B on a specific promoter. The residence time of individual Rap1 molecule at a target promoter was measured using this method to be largely 30 to 120 min. Confirming the results observed using other ChIP experimental systems [3,21,23], different genomic target loci show different Rap1 binding kinetics. Interestingly, a higher level of transcriptional activation was associated with stable, long-term Rap1 binding, whereas short term, rapid turnover binding (referred to as "treadmilling") is associated with low transcript levels. This work thus highlighted that the micro-scale stability of TF binding, rather than the cumulative occupancy of TF binding, is indicative of TF function. The authors proposed that a molecular clutch mechanism allows the switch between "treadmilling" mode and long-term binding mode [4]. Note that this "treadmilling" short-term interaction is distinct from the "hit-and-run" transient interaction [3], because the "treadmilling" refers to rapid turnover of binding at a particular loci, which could be detected by ChIP-Seq as a stable binding event.

Recent advances in single molecule tracking technology have provided better estimation of the residence time of a single TF on its target promoters. In a recent study, two key pluripotency regulators, Sox 2 and Oct4, were monitored using single molecule tracking in mouse embryonic stem cells [1]. Sox 2 was found to persist at an active transcription site for only $\sim 12$ s for specific binding. Similarly, p53 and GR have been visualized in live cell nuclei of human cell lines [2]. It was shown that the dwell times of p53 and GR within transcription domains are up to $\sim 15$ s for p53 and $\sim 25$ s for GR, associated with sequence-specific binding. Such highly transient binding suggests that the subset of occupied promoters will vary at the seconds scale. This would lead to transcriptional bursting of genes when there are only a few molecules of a TF present and a large number of promoters to activate, similar to the concept put forward in the "hit-and-run" model for bZIP1 in plants [3].

These transient but specific TF-target bindings measured in tens of seconds is different from the much shorter non-specific binding of TFs during their search for targets (Fig. 2C) [40]. For example, Sox 2 was detected to have a 0.8 s residence time for non-specific short-lived binding [1]. Similarly, the dwell times of p53 and GR outside of the transcriptionally active region is about only 3-4 seconds, considered to be associated with the target searching [2]. Different TFs can adopt different target searching strategies, ranging from exploring the whole nucleus to sampling a local region extensively [41].

In addition to single molecule tracking and competition ChIP, more techniques for studying TF kinetics are reviewed in [42]. The single molecule tracking method is powerful in detecting TF dynamics of single molecule in vivo. However, target genes for a TF-of-interest cannot be identified using the single molecule tracking method. By contrast, time-series ChIP-based method allows the identification of target genes bound by a specific TF and further investigation of the functional implications of such binding. However, ChIP-based methods lack the resolution for single molecules. A combination of different methods may provide a more comprehensive understanding of TF dynamics. All the time-course whole-genome studies on TF dynamic behavior discussed in this review are summarized in Table 1 with their experimental methods listed.

\section{Concluding remarks}

Dynamic binding of TFs to targets mediates complex GRNs in response to stimuli. Research in this direction has been facilitated by the development of high-throughput sequencing, single molecule tracking methods, and network reconstruction algorithms. We have begun to gain 
1 insights into how temporal modes of TF binding regulate dynamic gene expression. For example, 2 transient binding may enable a TF to activate multiple targets for a quick response in the GRN 3 [3]. It also seems that the same TF may bind to different targets with different dynamic behaviors, 4 and that the same temporal TF binding pattern may lead to different transcriptional outputs for 5 different targets. This highlights the possible influences of other factors. One recurrent theme 6 across all species and transcription models is that co-regulating TF partners play a big role in 7 determining the specific regulation of target genes. Thus, to explain the temporal behavior of a 8 GRN, it will be necessary to assay multiple TFs that are known or suggested to work together. 9 Another factor that may impact transcriptional output is the local chromatin landscape. Epigenetic modifications have been extensively profiled [20,43], adding another layer of information to our understanding of GRNs. For example, it was suggested that DNA demethylation and transcription factor binding interact to regulate fruit ripening in tomato [44]. In addition, dynamic DNA methylation changes have been reported during the stress response in Arabidopsis [45]. We expect that in the future, integrating temporal -omics datasets from multiple TFs and also epigenetic players will lead to a better understanding of the dynamic regulation of GRNs. Finally, minutes, hours, or days for long-term in planta physiological changes. Advances in network 18 modeling methods such as multiscale modeling [46] will hopefully integrate data gathered from 19 different time scales into a dynamic model that will allow a holistic understanding on how plants 20 respond to the environmental and developmental cues. 
Box 1 Gene regulatory network: A gene regulatory network is an abstracted model of gene regulation where nodes are genes and the edges indicate their interactions (Fig. 1). The interaction between genes can be causal regulation (e.g., TF to target genes, miRNA to target mRNA), or expression correlation, or physical interaction (e.g., protein-protein interactions, protein-DNA interactions). Time-series data is especially useful for learning and predicting causal interactions between TFs and target genes. A causal gene regulatory network can be inferred from time-series expression data [5], and a review of these approaches is found in [8]. Protein-protein interactions and protein-DNA interactions can further assist the reconstruction of gene regulatory networks from time-series expression data [47,48]. A gene regulatory network consists of a number of basic network motifs (a simple pattern of gene interactions that is repeated many times in the network). Certain network motifs are significantly enriched in gene regulatory networks, for example, the feed forward loop [49]. For a comprehensive review on recurrent network motifs please see [50]. Visualization of such dynamic gene regulatory networks in static images remains a challenge, and current studies code the dynamic behaviors of nodes and edges by different visual effects like color and line style, as shown in [21,27].

Box 2 Transient binding of a TF: What is the consequence of transient binding of a TF? The answer depends on the context. In plants, the hit-and-run model proposed for bZIP1 in Arabidopsis [3] suggests that the transient binding of bZIP1 to its targets enables rapid and transient nutrient signaling. In animals, the transient binding of GR and p53 to their binding sites, visualized by single molecule tracking technology, has also been proposed to enable transcriptional bursting of target genes [2]. By contrast, the molecular clutch model proposed for Rap1 in yeast [4] suggests that short-term binding, referred to as "treadmilling", leads to low transcript level, whereas long-term binding leads to high level activation of target genes. 
1 Table 1: Studies on the dynamic patterns of TFs.

\begin{tabular}{|c|c|c|c|}
\hline Organism & Time scale & Method & Reference \\
\hline Arabidopsis & $\begin{array}{l}0,0.25,0.5,1,4, \\
24 \mathrm{hr}\end{array}$ & ChIP-seq & [23] \\
\hline Arabidopsis & $\begin{array}{l}\text { Dark, 30min light, } \\
\text { 3hr light, } 24 \mathrm{hr} \text { light }\end{array}$ & DNase sequencing & [27] \\
\hline Arabidopsis & $\begin{array}{l}1 \mathrm{~min}, 5 \mathrm{~min}, 30 \mathrm{~min} \text {, } \\
1 \mathrm{hr}, 5 \mathrm{hr}\end{array}$ & ChIP-seq & [3] \\
\hline Drosophila & $\begin{array}{l}2 \mathrm{hr} \text { window up to } 12 \\
\mathrm{hrs}\end{array}$ & ChIP-chip & [22] \\
\hline $\begin{array}{l}\text { Human cell } \\
\text { lines }\end{array}$ & Seconds & $\begin{array}{l}\text { Single molecule } \\
\text { tracking }\end{array}$ & {$[2]$} \\
\hline $\begin{array}{l}\text { Mouse } \\
\text { embryonic } \\
\text { stem (ES) } \\
\text { cells }\end{array}$ & Seconds & $\begin{array}{l}\text { Single molecule } \\
\text { tracking }\end{array}$ & {$[1]$} \\
\hline $\begin{array}{l}\text { Primary } \\
\text { mouse } \\
\text { dendritic } \\
\text { cells } \\
\end{array}$ & $0,0.5,1,2 \mathrm{hr}$ & HT ChIP-seq & {$[20]$} \\
\hline Yeast & $0,5,15,30,45 \mathrm{~min}$ & ChIP-chip & [21] \\
\hline Yeast & $\begin{array}{l}0,10,20,30,40,50 \\
60,90,120,150 \mathrm{~min}\end{array}$ & $\begin{array}{l}\text { Competition ChIP- } \\
\text { chip }\end{array}$ & {$[4]$} \\
\hline
\end{tabular}


Figure 1. Gene regulatory networks. A gene regulatory network is an abstracted model of gene regulation where the nodes represent genes and the edges indicate different types of interactions. A gene regulatory network consists of various basic network motifs.

\section{Figure 2. Multiple temporal scales of dynamic transcriptional control of gene regulatory}

networks. (A) Biological processes including developmental processes (flowering) and long-term responses to environmental factors (nutrient, pathogen and stress) can occur over days.

Underlying these physiological changes are the changes in gene regulatory network that can happen within minutes or hours after receiving the environmental and developmental cues. Four examples of dynamic gene expression are shown. (B) The driving force of the expression changes in dynamic gene regulatory network are the dynamic TF binding of their specific targets, which can happen in seconds, minutes, or hours. (C) Before a TF settles down on a specific target, it may scan the genome by multiple millisecond interactions to search for targets.

\section{Figure legend}


1 Chen, J. et al. (2014) Single-Molecule Dynamics of Enhanceosome Assembly in Embryonic Stem Cells. Cell 156, 1274-1285

2 Morisaki, T. et al. (2014) Single-molecule analysis of transcription factor binding at transcription sites in live cells. Nat. Commun. 5,

3 Para, A. et al. (2014) Hit-and-run transcriptional control by bZIP1 mediates rapid nutrient signaling in Arabidopsis. Proc. Natl. Acad. Sci. 111, 10371-10376

4 Lickwar, C.R. et al. (2012) Genome-wide protein-DNA binding dynamics suggest a molecular clutch for transcription factor function. Nature 484, 251-255

5 Krouk, G. et al. (2010) Predictive network modeling of the high-resolution dynamic plant transcriptome in response to nitrate. Genome Biol. 11, R123

6 An, D. et al. (2012) Transcriptome profiling of low temperature-treated cassava apical shoots showed dynamic responses of tropical plant to cold stress. BMC Genomics 13, 64

7 Yu, L. et al. (2012) Comparative transcriptome analysis of transporters, phytohormone and lipid metabolism pathways in response to arsenic stress in rice (Oryza sativa). New Phytol. 195, 97-112

8 Krouk, G. et al. (2013) Gene regulatory networks in plants: learning causality from time and perturbation. Genome Biol. 14, 123

9 Leivar, P. et al. (2009) Definition of Early Transcriptional Circuitry Involved in LightInduced Reversal of PIF-Imposed Repression of Photomorphogenesis in Young Arabidopsis Seedlings. Plant Cell Online 21, 3535-3553

10 Maekawa, T. et al. (2012) Conservation of NLR-triggered immunity across plant lineages. Proc. Natl. Acad. Sci. U. S. A. 109, 20119-20123

11 Torti, S. et al. (2012) Analysis of the Arabidopsis Shoot Meristem Transcriptome during Floral Transition Identifies Distinct Regulatory Patterns and a Leucine-Rich Repeat Protein That Promotes Flowering[C][W][OA]. Plant Cell 24, 444-462

$12 \mathrm{Li}, \mathrm{G}$. et al. (2014) Temporal patterns of gene expression in developing maize endosperm identified through transcriptome sequencing. Proc. Natl. Acad. Sci. U. S. A. 111, 7582-7587

13 Xin, M. et al. (2013) Dynamic Expression of Imprinted Genes Associates with Maternally Controlled Nutrient Allocation during Maize Endosperm Development[W][OPEN]. Plant Cell 25, 3212-3227

$14 \mathrm{Yu}, \mathrm{K}$. et al. (2012) Transcriptome changes during fruit development and ripening of sweet orange (Citrus sinensis). BMC Genomics 13, 10

15 Troncoso-Ponce, M.A. et al. (2011) Comparative deep transcriptional profiling of four developing oilseeds. Plant J. 68, 1014-1027

$16 \mathrm{Wu}$, S.-H. (2014) Gene Expression Regulation in Photomorphogenesis from the Perspective of the Central Dogma. Annu. Rev. Plant Biol. 65, 311-333

17 Forde, B.G. (2014) Nitrogen signalling pathways shaping root system architecture: an update. Curr. Opin. Plant Biol. 21, 30-36

18 Péret, B. et al. (2011) Root developmental adaptation to phosphate starvation: better safe than sorry. Trends Plant Sci. 16, 442-450

19 Hoson, T. (2014) Plant Growth and Morphogenesis under Different Gravity Conditions: Relevance to Plant Life in Space. Life Open Access J. 4, 205-216

20 Garber, M. et al. (2012) A High-Throughput Chromatin Immunoprecipitation Approach Reveals Principles of Dynamic Gene Regulation in Mammals. Mol. Cell 47, 810-822

$21 \mathrm{Ni}$, L. et al. (2009) Dynamic and complex transcription factor binding during an inducible response in yeast. Genes Dev. 23, 1351-1363

22 Zinzen, R.P. et al. (2009) Combinatorial binding predicts spatio-temporal cis-regulatory activity. Nature 462, 65-U72

23 Chang, K.N. et al. (2013) Temporal transcriptional response to ethylene gas drives growth hormone cross-regulation in Arabidopsis. Elife 2, e00675 
24 O'Geen, H. et al. (2010) Using ChIP-seq technology to identify targets of zinc finger transcription factors. Methods Mol. Biol. Clifton NJ 649, 437-455

25 Fujisawa, M. et al. (2013) A Large-Scale Identification of Direct Targets of the Tomato MADS Box Transcription Factor RIPENING INHIBITOR Reveals the Regulation of Fruit Ripening. Plant Cell 25, 371-386

26 Monke, G. et al. (2012) Toward the identification and regulation of the Arabidopsis thaliana ABI3 regulon. Nucleic Acids Res. 40, 8240-8254

27 Sullivan, A.M. et al. (2014) Mapping and Dynamics of Regulatory DNA and Transcription Factor Networks in A. thaliana. Cell Rep. 8, 2015-2030

28 Marchive, C. et al. (2013) Nuclear retention of the transcription factor NLP7 orchestrates the early response to nitrate in plants. Nat. Commun. 4 ,

29 Jakoby, M. et al. (2002) bZIP transcription factors in Arabidopsis. Trends Plant Sci 7, 10611

30 Baena-González, E. et al. (2007) A central integrator of transcription networks in plant stress and energy signalling. Nature 448, 938-942

31 Hill, D.E. et al. (1986) Saturation mutagenesis of the yeast his3 regulatory site: requirements for transcriptional induction and for binding by GCN4 activator protein. Science 234, 451-7

32 Eadara, J.K. et al. (1996) Chromatin Structure and Factor Site Occupancies in an in VivoAssembled Transcription Elongation Complex. Nucleic Acids Res. 24, 3887-3895

33 McNally, J.G. (2000) The Glucocorticoid Receptor: Rapid Exchange with Regulatory Sites in Living Cells. Science 287, 1262-1265

34 Schaffner, W. (1988) Gene regulation. A hit-and-run mechanism for transcriptional activation? Nature $336,427-8$

35 Consortium, T.E.P. (2004) The ENCODE (ENCyclopedia Of DNA Elements) Project. Science 306, 636-640

36 Foley, J.W. and Sidow, A. (2013) Transcription-factor occupancy at HOT regions quantitatively predicts RNA polymerase recruitment in five human cell lines. BMC Genomics 14,720

37 Heyndrickx, K.S. et al. (2014) A Functional and Evolutionary Perspective on Transcription Factor Binding in Arabidopsis thaliana. Plant Cell 26, 3894-3910

38 Pfeiffer, A. et al. (2014) Combinatorial complexity in a transcriptionally centered signaling hub in Arabidopsis. Mol. Plant 7, 1598-1618

39 Zhang, Y. et al. (2013) A Quartet of PIF bHLH Factors Provides a Transcriptionally Centered Signaling Hub That Regulates Seedling Morphogenesis through Differential Expression-Patterning of Shared Target Genes in Arabidopsis. PLoS Genet 9, e 1003244

40 Hager, G.L. et al. (2009) Transcription Dynamics. Mol. Cell 35, 741-753

41 Izeddin, I. et al. (2014) Single-molecule tracking in live cells reveals distinct target-search strategies of transcription factors in the nucleus. eLife 3,

42 Mueller, F. et al. (2013) Quantifying transcription factor kinetics: At work or at play? Crit. Rev. Biochem. Mol. Biol. 48, 492-514

43 Wollmann, H. et al. (2012) Dynamic Deposition of Histone Variant H3.3 Accompanies Developmental Remodeling of the Arabidopsis Transcriptome. PLoS Genet. 8,

44 Zhong, S. et al. (2013) Single-base resolution methylomes of tomato fruit development reveal epigenome modifications associated with ripening. Nat. Biotechnol. 31, 154-159

45 Dowen, R.H. et al. (2012) Widespread dynamic DNA methylation in response to biotic stress. Proc. Natl. Acad. Sci. 109, E2183-E2191

46 Meier-Schellersheim, M. et al. (2009) Multiscale modeling for biologists. Wiley Interdiscip. Rev. Syst. Biol. Med. 1, 4-14

47 Gitter, A. et al. (2013) Linking the signaling cascades and dynamic regulatory networks controlling stress responses. Genome Res. 23, 365-376 
148 Wise, A. and Bar-Joseph, Z. (2015) cDREM: Inferring Dynamic Combinatorial Gene

2 Regulation. J. Comput. Biol. 22, 324-333

349 Milo, R. et al. (2004) Superfamilies of Evolved and Designed Networks. Science 303, 1538$4 \quad 1542$

550 Alon, U. (2007) Network motifs: theory and experimental approaches. Nat. Rev. Genet. 8 , $6 \quad 450-461$ 


\section{Response to Reviewers}

Li et al., "From milliseconds to lifetimes: Tracking the dynamic behavior of transcription factors in gene networks"

**For the line numbers referenced in this document, please use the inside line number in the manuscript, not the outside line number generated by the submission system.**

\section{Reviewer 1}

The authors of this review propose to discuss progress in profiling and modeling the dynamics of transcriptome changes over time, followed by discussion of mechanisms regulating these dynamics. Finally, they review cutting edge techniques for monitoring transcription factor (TF) dynamics. These subjects are timely and relevant to the forefront of plant genomics/systems biology. However, there are gaps in the narrative and the manuscript is, in my opinion, too short to do justice to the subject. I recommend the following areas be addressed before publication is considered:

1. The section on dynamic behavior of individual TFs is unclear and lacks mention of some relevant techniques. Table 1 lists experimental methods to study dynamic TF regulation, according to its title. However, it appears more to be a list of example studies investigating TF dynamics. Certain techniques are duplicated within the table, whilst others are missing. The authors might refer to a recent review by Mueller et al (doi:10.3109/10409238.2013.833891). Moreover, the table is not referred to in the main text.

*Editor Note: I agree with the Reviewer about Table 1. Currently, it is not a list of methods, but, as the Reviewer pointed out, a list of studies that have been done. If you want to keep the table as is, I suggest modifying the legend. However, I do think it would be quite useful to have a table that really did cover different methods, particularly as I felt the main text was vague on this question.

Author response: For a comprehensive review of different methods of studying TF kinetics, we directed the audience to Mueller et al (reference 42). We modified the legend of Table 1 so that the dynamic TF studies are listed according to their experimental design (time scale) and experimental technique. We added a reference to this table in the text.

2. A relatively large proportion of the text is devoted to a recent study by the authors' own lab, which investigated the hit and run model of transcriptional activation. Whilst this is an interesting and promising line of investigation, it seems inappropriate to devote so much discussion to the results of a single paper.

*Editor Note: I think if you expand on the other areas, the overall balance of the piece will be fine.

Author response: We have expanded on the other areas and shortened the text on the hit-and-run paper.

3. In the Conclusion the authors propose that epigenetic regulation may be a significant factor in dynamic TF regulation. At least two relevant studies exist that should be included here. The first demonstrates the influence of dynamic DNA methylation on TF binding and fruit ripening in tomato (doi:10.1038/nbt.2462). The second describes genome wide DNA methylation changes that correlate with gene expression changes in pathogen challenged plants (doi:10.1073/pnas.1209329109).

Author response: We have expanded our conclusion section based on these two new references (Zhong et al., 2013 [Reference 44] and Dowen et al, 2012 [Reference 45]).

4. The importance of combinatorial effects of TFs (i.e. binding of the same target genes) is discussed. No mention is made of recent papers that specifically explore combinatorial binding of plant TFs, for example from the Vandepoele lab (doi:10.1105/tpc.114.130591). Moreover, there is no discussion of data produced by the ENCODE and modENCODE, both of which analyze combinatorial TF interactions in detail and present differences to those identified in plants. There are many relevant papers; the authors might start with doi:10.1186/1471-2164-14-720. 
Author response: We have expanded our discussion on the combinatorial effect of TFs based on papers recommended by the reviewer (ENCODE project [Reference 35], Foley and Sidow 2013 [Reference 36] and Heyndrickx et al., 2014 [Reference 37]).

5. The section on global dynamics of TF binding would benefit from a more detailed examination of work from the Bar-Joseph lab. They have made advances in time series modeling beyond those described in Chang et al. 2013 (discussed in the manuscript). These begin to integrate protein-protein interactions into the modeling process. Work by a number of labs supports the idea that heterogeneous TF interactions influences DNA binding activity. This would further link to the discussion of combinatorial TF behavior, above.

Author response: In the revised manuscript, we have incorporated publications from Bar Joseph lab (Gitter et al., 2013 on sDREM [ref 47] and Wise et al., 2014 cDREM [ref 48]) to comment on the progress on time series modeling (Box 1, line 10). However, these papers are about modeling timeseries gene expression data, not time-series TF binding data as Chang et al., 2013 (ref 23). Therefore, these papers are discussed in Box 1 in the context of reconstructing dynamic gene regulatory networks. We also cite a review Krouk 2013 (ref 8) that reviews the modeling of dynamic networks based on time-series transcriptome data (ref 5).

6. The manuscript starts by asserting that dynamic responses of gene regulatory networks (GRNs) occur over different time scales. However, some points supporting this are not well founded. It is stated that abiotic stimuli cause changes in GRNs within minutes, whilst biotic stimuli cause changes over hours. I am unaware of any study investigating GRN changes in response to biotic stimuli on a second or minute timescale. To do so would require single cell/small cell number analyses, or protoplast infection studies, due to the nature of initial pathogen infection. Consequently I think that this contrast is not valid. The authors need either to present evidence or clarify their argument.

Author response: We agree with the reviewer and have edited this sentence to read: "This is likely due to the nature of pathogen infection - that it is difficult to measure response in minutes unless single cells or protoplasts are challenged with pathogen." (see Page 2 line 22). 


\section{Reviewer 2}

This review by Li et al describes recent progress in the study of dynamic gene regulatory networks, especially in plants. The authors highlight gene expression changes in response to external stimuli, discussing how the dynamics of gene regulatory networks is regulated. A major focus of this review are the temporal changes in transcription factor (TF) binding to the target sequences in the genome, which has been investigated by recent studies including their previous report describing a "Hit-and-Run" model. They also address studies in the other organisms and discuss the similarities with plants regarding temporal gene expression and TF binding patterns. In the last portion of the piece, the authors review the kinetics of TF binding as measured by single-molecule imaging technology. The topic of this review, which encompasses the dynamics of gene expression and transcription factor binding is important and useful for understanding recent progress in this field. However, there are several areas in which revision would greatly improve the piece.

1) The authors should take the time to more precisely define the meaning of key terms used in the manuscript. For example, it is often difficult to understand what the authors mean when using the word "dynamics", and whether the definition of "dynamics" is consistent throughout the piece.

Author response: We have examined the use of "dynamic" throughout the manuscript, and made edits to make it more informative and accurate.

2) On page 2, the authors lists the response to light exposure as an example of "rapid response that has occurred within one hour". On the other hand, they mention the biotic signals measured over the course of hours as the "long-term responses". The time-scale used to define rapid and long-term is confusing.

Author response: In this context, we defined response in minutes ( $\leq$ one hour) as rapid, and defined multiple hours to days as long-term response. We have added descriptive terms to define "rapid" and "long-term" in the related sentences (page 2 line 21), as requested by the reviewer and editor, to make it clear.

3) The authors mention, "By contrast to these rapid responses to abiotic signals... are possibly controlled by multiple feed-back loops to restrain the scale and scope of the defense response", and cite Maekawa, T. et al. (2012) PNAS. 109. Is this citation correct? This paper describes an evolutionally conserved resistance mechanism. Multiple feed-back loops are not discussed in this paper

Author response: The PNAS paper by Maekawa et al., 2012 (ref 10) showed a MLA1-dependent gene cluster, whose expression level increases in response to biotic stress at 12 and 18 hours but goes down in 24 hours. Although it is not discussed in Maekawa et al, we think that this transiently up-and-down expression pattern suggests feed-back loops to restrain the response. We have revised the manuscript to make this point more clear (Page 2, Line 24-28).

4) Reference [20] and [21] discuss regulatory mechanisms for temporal gene expression patterns. However, the authors claim "little has been said about the underlying regulatory mechanisms."

*Editor Note: See my change to this sentence to address this point.

Author response: We have changed the sentence according to the editor's suggestion (Page 2, Line 43).

5) The authors only give the example of NLP7 for explaining the molecular mechanisms regulating the timing of TF-target interaction, which could result in misleading generalizations. Also, how the binding timing of NLP7 is controlled is not adequately described.

Author response: We expanded on the mechanism of controlling NLP7-targets interaction - by controlling the nuclear localization of NLP7 (Marchive et al 2013, ref 28). We also added that this is one possible mechanism for regulating temporal TF-target interactions, other than a generalized 
mechanism. In addition, we moved the paragraph of NLP7 down in the section to make the flow better. (Page 3, Line 44).

6) On page 3 the sentence "Below, we review some recent findings on dynamic signaling in plants..." is not clear to me. What is "dynamic signaling including dynamic binding of TFs to their genome-wide targets"?

Author response: This sentence was removed according to the editor's suggestion.

7) The sentence "the dynamic binding of EIN3 leads to four distinct waves of transcriptional response to ethylene." is not clear to me. Please explain in detail the "four distinct waves of transcription responses".

Author response: We have expanded on the details of the four waves of transcriptional response. (Page 3, Line 19).

8) The sentence "The EIN3 time-course study employed ChIP-based whole genome profiling..." seems strange because most of the studies cited in this review employ ChIP.

Author response: This sentence was edited according to the editor's suggestion (Page 3, line 31-32).

9) On page 4, although the authors mention that "many parallels can be drawn between the dynamic gene networks in plants and animals", they also say "Studies from plants and animals reach a few common conclusions". These two sentences are inconsistent and it is not clear to me whether plants and animals share many common features or not.

Author response: We have reworded the second sentence to be more clear (Page 5, Line 29).

10) The authors say "1) the same dynamic TF binding can lead to different gene expression patterns". This sentence does not make sense to me. What is "the same dynamic TF binding"? Also, what is the "teamwork between TF partners" in the sentence of \#4 conclusion? The authors should explain this more clearly in concrete terms. Citations should be added to each conclusion (\#1-4).

Author response: This sentence means that the same temporal binding pattern of a specific TF on gene A and gene B may not result in the same expression pattern across time. For example, gene A might be induced early and transiently, while gene B might show a late and steady induction. We edited the related sentences to make it clear (Page 5, line 29). We also reworded and cited the supporting paper for points 1-4, as suggested by the reviewer (Page 5, line 20-33, reference 3,20$22,27,37)$.

11) On page 5, the authors discuss "The distinct advantage in studying these dynamics in plants.....the animal and yeast studies are typically performed in cell-lines or single cell setups". The authors should explain to the reader more specifically why they think that unicellular organisms or human cell lines are not good systems in which to TF binding dynamics and gene regulation networks, especially given that they have acknowldeged the similarities between these systems and plants.

Author response: We have reworded this part according to the reviewer's comments (page 5, line 34$38)$.

12) The following sentences should be clarified and made more concise: "The binding pattern is therefore determined.....the same TF molecule stays on the promoter from 5-30 minutes?".

Author response: We have reworded these sentences according to the reviewer's comments (Page 5, line 45-48).

13) The authors may be confusing rapid TF binding turnover with transient TF binding. These are distinct concepts and phenomena. The relevant section is:

"Note that this "treadmilling" short-term interaction is different from the "hit-and-run" transient interaction, because the latter does lead to a substantial change in transcript level."

I agree that these two phenomena are distinct, but it is not because of the transcriptional outcome. It's because "treadmilling" is rapid turnover of binding at a particular location, which is not necessarily transient (the treadmilling interaction might be very constant). 
Author response: We have reworded the part comparing treadmilling and hit-and-run according to the reviewer's suggestion (Page 6, lines 13-15).

Related to the comment above, the sentences "The Hit-and-run model identified for bZIP1 in plants, also explains the continued activation of targets through...." should be removed. This statement does not make sense in the context of this paragraph, which focuses on the residence time of TFs as determined by single molecule tracking technologies.

Author response: This sentence has been removed.

14) On page 6 , the paragraph containing:

"The above-mentioned transient but specific TF-target binding measured in... For a review on the nonspecific dynamic TF binding for target searching, please refer to..."

should be rewritten to eliminate awkward wording.

Author response: We have rewritten this part according to the reviewer's and editor's suggestion (Page 6, Lines 29-35).

15) The discussion of the advantages and disadvantages of single-molecule technology and ChIP is not balanced. The authors should make an effort to present a more balanced review, or state explicitly that portion of the review is their opinion.

Author response: We have rewritten this part to present a more balanced review of the two methods (Page 6, lines 37-44).

16) The Conclusion and Perspective section focuses too much on the authors' own findings, which could create a perception of reduced credibility.

Author response: We have reduced the text on "hit-and-run" in the conclusion and perspective section (the current "concluding remarks"). 


\section{Major changes}

Li et al., "From milliseconds to lifetimes: Tracking the dynamic behavior of transcription factors in gene networks"

\section{Section "Dynamic GRNs"}

Page 2 line 22-24: We have edited the sentences on the contrast of the time scale between biotic stress responses and abiotic stress responses.

Page 2 line 24-28: We have revised the manuscript to include more details from the MLA1 study (Maekawa et al., 2012, ref 10), and mark the distinction between the study and our interpretation of the study.

\section{Section "Global dynamic pattern of TF binding”}

Page 3 line 3-10: We added more details and take-home messages from the Zinzen et al study(ref 22) on multiple TF binding data and spatial-temporal expression modeling.

Page 3 line 13-29: We edited the paragraph on EIN3 study (Chang et al ref 23) to add details and to stress the important take-home messages.

Page 3 line line 44-50: We expanded on the mechanism of controlling NLP7-targets interactions. In addition, we also moved the paragraph about NLP7 down in the section to make the flow better.

Page 4 line 5-16: We have shortened the text on the hit-and-run bZIP1 paper (ref 3), and expanded on the other areas to achieve a better balance.

Page 4 line 21-33: We have expanded our discussion on the combinatorial effect of TFs.

Page 5 line 20-33: We have edited the discussion on the common features of dynamic GRNs across plants and animals to make it easier to understand for general audience.

Page 5 line 33-38: We have edited the discussion on the comparison of animal/yeast cell system v.s. plant systems of whole organism.

\section{Section "Dynamic behavior of individual TFs in the nucleus"}

Page 6 line 13-15: We have reworded the part comparing treadmilling and hit-and-run according to the reviewer's suggestion.

Page 6 line 38-44: We have rewritten the part on comparing the two methods, ChIP and single molecule tracking, to present a more balanced view.

\section{Section "Concluding remarks"}

Page 7 line 9-13: we have expanded our conclusion section based on the two new references (ref 44 and ref 45) on time-course studies of DNA methylation.

\section{Text boxes}

We switched the order of text box 1 and text box 2 . 
Text box 1 line 10: We incorporated publications from Bar Joseph lab (Gitter et al., 2013 on sDREM and Wise et al., 2014 cDREM, ref 47 and 48) to comment on the progress on time series modeling.

Text box 1: added figure 1 .

\section{Table}

Table 1: We modified the legend of Table 1.

\section{Figure legend}

Figure 1: We added figure 1.

Figure 2: We expanded the legend of figure 2. 
Time series transcriptome

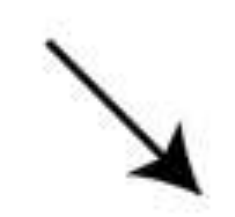

Protein-protein interaction TF-target interaction
Gene expression correlation

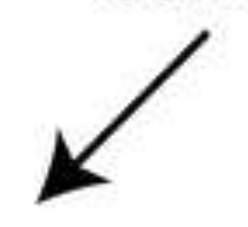

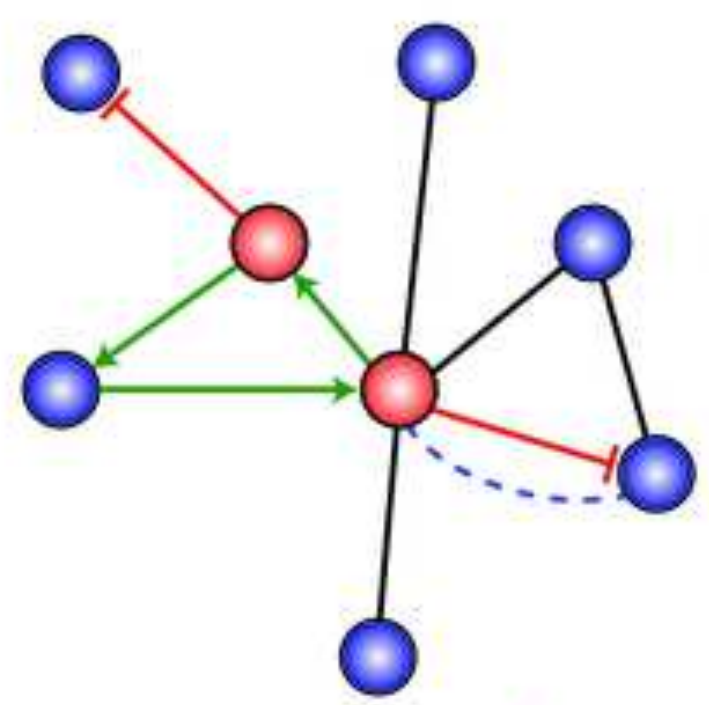

basic motif

Otranscription factors

Oother genes
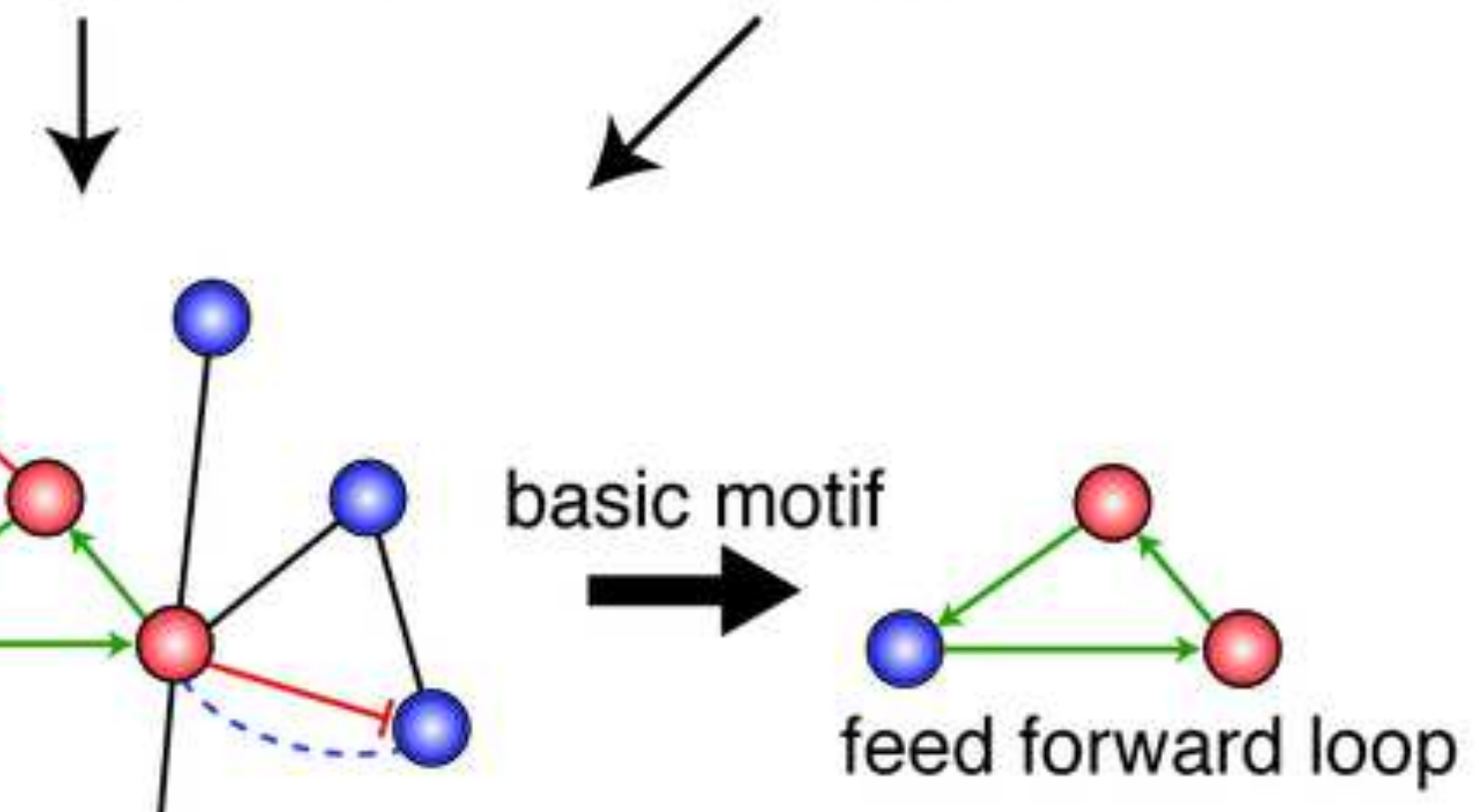

$\rightarrow$ predicted TF activation

$\longrightarrow$ predicted TF repression

....... gene experssion correlation

— protein-protein interaction 


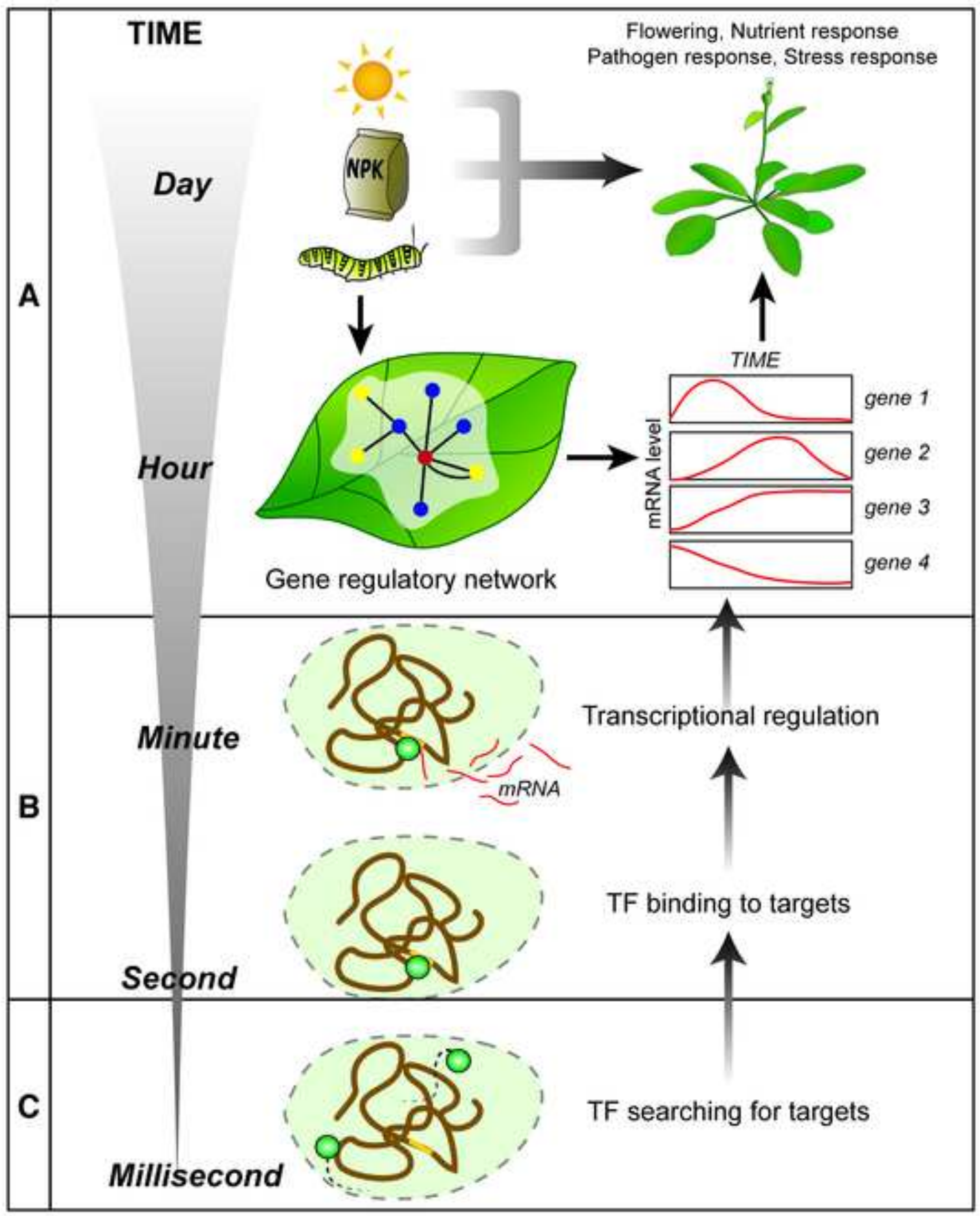

